\title{
Efficacy of Combined Phototherapeutic Keratectomy, Wavefront-optimized Photorefractive Keratectomy and Corneal Collagen Crosslinking in the Management of Progressive Keratoconus over One-year Follow-up: Modified Athens Protocol
}

\author{
Mohammad M Shehadeh ${ }^{1}$, Jamal Qaddumi ${ }^{2}$, Mohammad T Akkawi ${ }^{3}$, Dima Sadi ${ }^{4}$, Ahmad R Soboh ${ }^{5}$, Deyab R Khloof ${ }^{6}$, \\ Ammar A Aghbar ${ }^{7}$
}

\begin{abstract}
Purpose: Keratoconus is a degenerative, ectatic corneal disorder. Recently, corneal crosslinking (CXL) as a stabilizing procedure has been combined with other procedures to improve visual and refractive outcomes as in Athens and Cretan protocols. The aim of the study is to present the efficacy and safety of a modified Athens protocol over 1-year follow-up.

Materials and methods: The study is a retrospective descriptive one with a total of 43 eyes (36 patients) with progressive keratoconus who underwent combined transepithelial phototherapeutic keratectomy (PTK), partial wavefront-optimized photorefractive keratectomy, and corneal collagen CXL. Visual, refractive, pachymetric and keratometric preoperative and postoperative follow-up data at 1 month, 3 months, 6 months, and 1 year were taken from patients' medical records.

Results: The mean uncorrected distance visual acuity (UDVA) improved from $(0.33 \pm 0.19)$ preoperatively to $(0.64 \pm 0.27)$ at 1 year postoperatively and the mean corrected distance visual acuity $(C D V A)$ from $(0.62 \pm 0.21)$ to $(0.80 \pm 0.20)$. At 1-year postoperative visit, the mean spherical and cylindrical values changed significantly from $(-2.10 \pm 2.45)$ to $(-0.34 \pm 2.26)$ and from $(-3.50 \pm 1.85)$ to $(-1.23 \pm 1.02)$, respectively. The mean steep and flat $K$ readings significantly decreased from $(50.97 \pm 4.46)$ to $(47.58 \pm 5.61)$ and from $(47.08 \pm 4.02)$ to $(44.84 \pm 4.47)$, respectively. The mean $K_{\max }$ also decreased from $(56.27 \pm 6.40)$ preoperatively to $(51.22 \pm 7.94)$ postoperatively. The thinnest corneal thickness mean was $(462.49 \pm 36.17 \mu \mathrm{m})$ preoperatively and $(388.21 \pm 56.64 \mu \mathrm{m})$ postoperatively.

Conclusion: Our modified Athens protocol has shown to be safe and efficacious in the management of progressive keratoconus. Impressive improvements in visual, refractive, and keratometric values were noted. This approach offers a more tissue saving protocol than the original Athens protocol with less alteration to the surface of the cornea, which may offer more predictability and less refractive surprises.

Keywords: Athens protocol, Cretan protocol, Crosslinking, Efficacy, Keratoconus.

International Journal of Keratoconus and Ectatic Corneal Diseases (2019): 10.5005/jp-journals-10025-1173
\end{abstract}

\section{INTRODUCTION}

Keratoconus is a degenerative ectatic disorder characterized by progressive corneal protrusion and paracentral corneal thinning leading to impairment in visual function. ${ }^{1}$ It is considered the most common primary corneal ectatic disease. ${ }^{2}$ In the last 2 decades, photochemical crosslinking (CXL) has been developed to arrest disease progression, improve corneal keratometry, and reduce corneal irregular astigmatism. ${ }^{3}$ Crosslinking -plus procedures, which have been proposed by many ophthalmologists, proved to be effective in further improvement of the refractive errors associated with keratoconus. The Cretan and Athens protocols use the phototherapeutic keratectomy (PTK) to remove the epithelium and regularize the surface of the cornea. ${ }^{4}$ The Athens protocol makes an additional normalization of the corneal surface and partial correction of refractive errors by topography-guided photorefractive keratectomy (topo-guided PRK). ${ }^{5-11}$ In this article, the results of our modified Athens protocol, which is PTK, partial wavefront-optimized rather than topo-guided PRK, and CXL in the management of progressive keratoconus over 1-year follows-up are shown.
${ }^{1,3-7}$ Ophthalmology Department, An-Najah National University Hospital, Faculty of Medicine and Health Sciences, An-Najah National University, Nablus, Palestine

${ }^{2}$ Department of Nursing, Faculty of Medicine and Health Sciences, An-Najah National University, Nablus, Palestine

Corresponding Author: Mohammad M Shehadeh, Ophthalmology Department, An-Najah National University Hospital, Faculty of Medicine and Health Sciences, An-Najah National University, Nablus, Palestine, Phone: +970 595202800, e-mail: mohammad.sh@najah.edu How to cite this article: Shehadeh MM, Qaddumi J, Akkawi MT, et al. Efficacy of Combined Phototherapeutic Keratectomy, Wavefrontoptimized Photorefractive Keratectomy and Corneal Collagen Crosslinking in the Management of Progressive Keratoconus over One-year Follow-up: Modified Athens Protocol. Int J Kerat Ect Cor Dis 2019;8(1):12-16.

Source of support: This work has been entirely made by the efforts and financial support of the authors. No fund or financial assistance has been provided from other resources

Conflict of interest: None

o The Author(s). 2019 Open Access This article is distributed under the terms of the Creative Commons Attribution 4.0 International License (https://creativecommons. org/licenses/by-nc/4.0/), which permits unrestricted use, distribution, and non-commercial reproduction in any medium, provided you give appropriate credit to the original author(s) and the source, provide a link to the Creative Commons license, and indicate if changes were made. The Creative Commons Public Domain Dedication waiver (http://creativecommons.org/publicdomain/zero/1.0/) applies to the data made available in this article, unless otherwise stated. 


\section{Materials and Methods}

This is a retrospective descriptive study of a total of 43 eyes of 36 patients (median age 26 years, range 18-35) with progressive keratoconus grades I-III on Amsler-Krumeich classification. The data were collected retrospectively from the patients' records at the Department of Ophthalmology, An-Najah National University Hospital after the approval of the study by An-Najah National University IRB Committee. These patients underwent same-day PTK, partial wavefront-optimized PRK, and CXL between November 2015 and December 2016.

The diagnosis of progressive keratoconus was based on slit lamp examination, corneal tomography, keratometry, corneal pachymetry, and subjective refraction. Preoperative data and follow-up data at 1, 3, 6 months, and 1-year postoperatively were taken from the patients' medical records.

\section{Measurements and Analysis}

Preoperative and postoperative evaluation included uncorrected distance visual acuity (UDVA) and corrected distance visual acuity (CDVA) in decimals. Cylindrical and spherical values were measured using subjective refraction. Flat, steep, mean, and maximum keratometric values $\left(K 1, K 2, K_{m}\right.$, and $\left.K_{\max }\right)$ and pachymetric (thinnest corneal thickness) corneal measurements using Pentacam Scheimpflug imaging device (Pentacam, Oculus, Wetzlar, Germany).

Descriptive statistics were performed using the Statistical Package for Social Sciences (SPSS Statistics V.22.0, SPSS Inc., Chicago, Illinois, USA). We considered $p$ values less than 0.05 statistically significant. The visual acuity is represented in decimals and keratometric values in diopters (D). Results were reported as mean \pm standard deviation and range as minimum to maximum.

\section{Inclusion Criteria}

The criteria set out before the study were clinical diagnosis with progressive keratoconus and a minimum age of 18. Patients with systemic diseases (collagen vascular diseases and diabetes mellitus), previous eye surgery or corneal scar, chemical injury or delayed corneal epithelial healing, and pregnancy or lactation were excluded from the study. Progression of keratoconus was defined by an increase in $K_{\max } \geq 2 \mathrm{D}, \mathrm{K}_{\max }-K_{\min } \geq 1 \mathrm{D}, K_{\mathrm{m}} \geq 0.75 \mathrm{D}$, pachymetry $\geq 2 \%$ decrease in CCT, corneal apex power $>1 D$ and manifest refraction spherical equivalent (MRSE) $>0.5 \mathrm{D}$ over 6 months.

\section{Surgical Technique}

All procedures were performed at An-Najah National University Hospital by the same surgeon (MMS) under sterile conditions. Topical anesthesia was applied by proxymetacaine $\mathrm{HCL} 0.5 \%$ eye drops. Then, transepithelial PTK ablation at a depth of $50 \mu \mathrm{m}$ using an optical zone of $7 \mathrm{~mm}$ and transitional zone of $1.25 \mathrm{~mm}$ was used to remove the corneal epithelium, followed by up to $70 \%$ wavefrontoptimized PRK laser ablation; the degree of correction and the optical zone were modified so that the remnant stromal bed did not go below $350 \mu \mathrm{m}$. The optical zone ranged from $5.5 \mathrm{~mm}$ to 6.0 $\mathrm{mm}$. Subsequently, mitomycin C $0.02 \%$ solution was applied over the ablated corneal surface for 20 seconds using a cellulose sponge. It was followed by copious irrigation with $30 \mathrm{~mL}$ of balanced salt solution. Thereafter, riboflavin $0.1 \%$ sodium phosphate solution was instilled repeatedly every 3 minutes for 30 minutes. The surgeon used the blue light of the slit-lamp to monitor the presence of riboflavin in the anterior chamber and saturation of the corneal stroma. Corneal thickness was measured just before the ultraviolet light irradiation to ensure that the corneal thickness exceeded 400 $\mu \mathrm{m}$. If the thickness was below $400 \mu \mathrm{m}$, dextran-free hypotonic riboflavin was used to swell the cornea. The cornea was irradiated for 30 minutes by $3 \mathrm{~mW}$ ultraviolet-A light of mean $370 \mathrm{~nm}$ wavelength. At the end of the procedure, a bandage contact lens was applied, and it was removed around day 7 postoperatively when the epithelial layer was healed. Postoperative medications included topical gatifloxacin $0.3 \%$ four times daily for 7 days and dexamethasone $0.1 \%$ four times daily tapered over 3 weeks. The patients were instructed to protect their eyes from the natural light with sunglasses.

\section{Results}

\section{Visual Acuity Changes}

Preoperative and postoperative UDVA and CDVA are presented in Table 1. Both showed significant improvement 1 year after the procedure. Mean UDVA improved from $0.33 \pm 0.19$ to $0.49 \pm 0.25$ at 1 month. Further improvement to $0.61 \pm 0.28$ was noticed at 3 months. Minimal improvement to $0.64 \pm 0.27$ was noticed thereafter at 1 year.

Mean CDVA improved from $0.62 \pm 0.21$ to $0.68 \pm 0.22$ at 1 month. Additional improvement to $0.79 \pm 0.22$ was noticed at 3 months. Minimal improvement to $0.80 \pm 0.20$ was noticed thereafter at 1 year.

\section{Keratometric Outcomes}

The mean steep $K$ readings showed significant reductions from $50.97 \pm 4.46$ preoperatively to $48.65 \pm 4.95$ at 1 month postoperatively. Over the next year, follow-up showed minimal changes toward stability as following $47.97 \pm 4.96$ at 3 months, 47.68 \pm 5.24 at 6 months, and $47.58 \pm 5.61$ at 1 year. The flat $K_{\text {mean }}$ readings also showed significant reduction from $47.08 \pm 4.02$ preoperatively to $45.68 \pm 4.08$ at 1 month postoperatively, minor changes were noted at 3 months $45.30 \pm 4.13$ and up to 1 year $44.84 \pm 4.47$. The $K_{\text {mean }}$ readings exhibited significant improvement from $48.94 \pm$ 4.15 preoperatively to $47.11 \pm 4.41$ at 1 month postoperatively.

Table 1: Visual acuity data of participants over 1-year follow-up

\begin{tabular}{|c|c|c|c|c|c|}
\hline Parameters & Preoperative (43 eyes) & 1 month (43 eyes) & 3 months (43 eyes) & 6 months ( 37 eyes) & 1 year (34 eyes) \\
\hline \multicolumn{6}{|l|}{ UDVA } \\
\hline Mean \pm SD & $0.33 \pm 0.19$ & $0.49 \pm 0.25$ & $0.61 \pm 0.28$ & $0.64 \pm 0.26$ & $0.64 \pm 0.27$ \\
\hline Gain/loss & - & +0.16 & +0.28 & +0.31 & +0.30 \\
\hline \multicolumn{6}{|l|}{ CDVA } \\
\hline Mean \pm SD & $0.62 \pm 0.21$ & $0.68 \pm 0.22$ & $0.79 \pm 0.22$ & $0.79 \pm 0.21$ & $0.80 \pm 0.20$ \\
\hline Gain/loss & - & +0.06 & +0.17 & +0.17 & +0.20 \\
\hline
\end{tabular}

CDVA, corrected distance visual acuity; SD, standard deviation; UDVA, uncorrected distance visual acuity 
Changes were minimal at 3 months $46.63 \pm 4.41$ and reached $46.11 \pm 5.01$ at 1 year. $K_{\max \text { mean }}$ readings preoperatively were $56.27 \pm 6.40$ and showed a significant change to $51.50 \pm 8.24$ at 1 month postoperatively. Small changes were noticed over the next year $51.22 \pm 7.94$, as shown in Table 2 .

\section{Corneal Thickness}

As a result of laser ablation used during PTK and PRK, a significant reduction in the thinnest corneal thickness mean of $84.5 \mu \mathrm{m}$ was noticed at 1 month postoperatively. However, it slightly increased over the 1-year follow-up, as illustrated in Table 3.

\section{Refractive Outcomes}

A significant improvement in cylindrical and spherical equivalents was noticed at a 1-month visit, postoperatively. The mean changes of cylindrical and spherical values were (change: $+1.54 \pm 1.69$ ) and (change: $+1.86 \pm 3$ ), respectively, with gradual improvement over the subsequent visits, as shown in Table 4.

\section{Discussion}

Keratoconus is a progressive noninflammatory biomechanical weakness of corneal collagen which is characterized by thinning and steepening that leads to abnormal topographic findings and visual function impairment. ${ }^{1}$ Corneal cross linking was developed to provide corneal stability, halt progression of corneal ectasia, and reduce the refractive and keratometric irregularities. The Cretan protocol was introduced by Kymionis et al. They showed that transepithelial PTK to remove the corneal epithelium has a positive regularizing and smoothing effect on the corneal surface because it uses masking and neutralizing effect of the epithelium with minimal stromal ablation. A follow-up of 23 eyes over 24-56 months after PTK and CXL by Kymionis et al. revealed improvement in UDVA, CDVA, and keratometric readings without intraoperative or postoperative complications. ${ }^{4}$ However, Kanellopoulos and Binder described the management of corneal ectasia after LASIK with combined, sameday, topography-guided partial transepithelial PRK, and collagen
CXL: the Athens protocol. ${ }^{5}$ We believe that combining the PTK, which alters the topography of the ectatic cornea and the topoguided PRK, which also modifies the topography of the cornea may lead to unwanted refractive surprises in some cases. Furthermore, topo-guided PRK sacrifices more corneal tissue compared with the wavefront-optimized ablation. Therefore, in our modified Athens protocol, we use the effect of the transepithelial PTK (the Cretan protocol) to make some regularization of the keratoconic cornea with the advantage of minimal tissue ablation, and the wavefront-optimized PRK with additional tissue saving to decrease the refractive errors of the keratoconic patients. We believe that our modified Athens procedure will be beneficial as a rehabilitation procedure for patients with anisometropia, patients who want to decrease rather than to eliminate their dependency on glasses and patients who want to improve their uncorrected and corrected visual acuity. It should not be used as a refractive procedure for patients with high expectations who want to achieve spectacle independency, which may cause disappointment for these patients.

\section{Visual Acuity Changes}

Based on our results, there was a significant improvement in UDVA, starting at 1 month, continuing at 3 months and 6 months postoperatively with a noticeable positive gain, reaching an average gain of three lines at 1 year. At 12 months, 91.2\% of eyes gained at least one line $(47.1 \%$ of eyes gained $\geq 3$ lines and $26.5 \%$ of eyes gained $\geq 5$ lines). The same pattern of improvement was noted in CDVA: $70.6 \%$ of eyes gained at least one line, $41.2 \%$ of eyes gained $\geq 3$ lines, and $14.7 \%$ of eyes gained $\geq 5$ lines. Overall, the average gain was two lines at 1 year.

Two of 43 eyes reported a loss of one line in UDVA, yet gain in the CDVA was still obtained. Four of 43 had a loss of one line in the CDVA, with an improvement in the UDVA. One eye had a loss of two lines in both UDVA and CDVA. This may be attributed mostly to corneal haze. This complication has frequently been reported in some studies. It can be explained by the use of CXL and its physiological wound healing and corneal depth changes postoperatively with keratocyte loss. ${ }^{12}$ The patient might not

Table 2: Anterior keratometry $(K)$ measured by the Scheimpflug device

\begin{tabular}{llllll}
\hline Parameters & Preoperative (43 eyes) & 1 month (43 eyes) & 3 months (43 eyes) & 6 months (37 eyes) & 1 year (34 eyes) \\
\hline Steep $K(D)$ mean \pm SD & $50.97 \pm 4.46$ & $48.65 \pm 4.95$ & $47.97 \pm 4.96$ & $47.68 \pm 5.24$ & $47.58 \pm 5.61$ \\
Flat $K$ (D) mean \pm SD & $47.08 \pm 4.02$ & $45.68 \pm 4.08$ & $45.30 \pm 4.13$ & $44.88 \pm 4.15$ & $44.84 \pm 4.47$ \\
$K_{\text {mean }}(D)$ mean \pm SD & $48.94 \pm 4.15$ & $47.11 \pm 4.41$ & $46.63 \pm 4.41$ & $46.26 \pm 4.69$ & $46.11 \pm 5.01$ \\
$K_{\max }(D)$ mean \pm SD & $56.27 \pm 6.40$ & $51.50 \pm 8.24$ & $51.53 \pm 7.57$ & $51.27 \pm 7.55$ & $51.22 \pm 7.94$ \\
\hline
\end{tabular}

$\mathrm{D}$, diopters; SD, standard deviation

Table 3: Thinnest corneal thickness

\begin{tabular}{llllll}
\hline Parameters & Preoperative (43 eyes) & 1 month (43 eyes) & 3 months (43 eyes) & 6 months (37 eyes) & 1 year (34 eyes) \\
\hline $\begin{array}{l}\text { Thinnest corneal thickness } \\
\text { mean }\end{array}$ & $462.49 \pm 36.17$ & $378.05 \pm 48.95$ & $376.88 \pm 50.81$ & $386.51 \pm 54.02$ & $388.21 \pm 56.64$
\end{tabular}

mean \pm SD

SD, standard deviation

Table 4: Refractive outcomes

\begin{tabular}{llllll}
\hline Parameters & Preoperative (43 eyes) & 1 month (43 eyes) & 3 months (43 eyes) & 6 months (37 eyes) & 1 year (34 eyes) \\
\hline Sphere mean \pm SD & $-2.10 \pm 2.45$ & $-0.24 \pm 2.46$ & $-0.43 \pm 2.71$ & $-0.42 \pm 2.53$ & $-0.34 \pm 2.26$ \\
Cylinder mean \pm SD & $-3.50 \pm 1.85$ & $-1.96 \pm 1.25$ & $-1.74 \pm 1.54$ & $-1.29 \pm 1.17$ & $-1.23 \pm 1.02$ \\
\hline
\end{tabular}

SD, standard deviation 
require specific treatment or sometimes need flourometholone eye drops (low dose corticosteroids) with vitamin C. However, considering a stromal haze, an expectant normal finding post-CXL has been controversial. ${ }^{13}$

\section{Keratometric Changes}

Based on our keratometric results mentioned above, we found a statistically significant improvement in the steep, flat and steepest $\mathrm{K}$, noticing the major decline at 1 month postoperatively, with a minimal yet continuous decline at 3 months, 6 months, and 1 year. This is an anticipated result of corneal anterior surface normalization done by PTK and PRK.

\section{Pachymetric Changes}

The postoperative corneal thickness, demonstrated by the thinnest corneal thickness, experienced a great reduction, which would be expected due to tissue excimer ablation of the corneal stroma. At 1 month, due to the epithelization process occurring at the corneal cellular level, a nadir thinnest would still not be expected. It is noteworthy, however, to mention that the main reduction was at 3 months because of halted epithelization process and tissue shrinkage and remodeling. However, corneal thickness was increasing progressively after 3 months as a result of the stromal reflectivity and density modification between the anterior and intermediate part of the cornea associated by CXL. ${ }^{14}$

\section{Refractive Changes}

According to refractive outcomes, there were significant improvements in mean spherical and cylindrical values from $(-2.10 \pm 2.45)$ to $(-0.34 \pm 2.26)$ and $(-3.50 \pm 1.85)$ to $(-1.23 \pm 1.02)$, respectively, after 1-year follow-up. This would be mostly as a result of partial correction by PRK and the flattening effect of cross linking.

\section{Conclusion}

Based on our study, the majority of cases demonstrated potentially promising refractive, keratometric and topometric results over 1-year follow-up after the use of the combination of PTK, wavefrontoptimized PRK and CXL as a safe and effective therapeutic intervention in treating mild to moderate irregular keratoconic corneas. This combined approach is a viable option for keratoconic progression and improve corneal regularity and offer a temporary or permanent alternative to keratoplasty.

Additional studies for a longer period of follow-up may be warranted to confirm the efficacy and stability of cases treated with this combined approach.

\section{Declarations}

\section{Ethics Approval and Consent to Participate}

Ethical approval was granted by An-Najah National University, IRB ethical committee.

\section{Consent for Publication}

The manuscript does not contain any person's data in any form.

\section{Availability of Data and Material}

Based on the ethical approval from An-Najah National University, IRB ethical committee, data will be kept confidential, and we can only submit the findings.

\section{Authors' Contributions}

Mohammad M Shehadeh has made substantial contributions to the conception and design of the study, acquisition of data, analysis and interpretation of the data, and drafting of the manuscript. Jamal Qaddumi has made substantial contributions to data collection and interpretation, and drafting the manuscript. Mohammad T Akkawi has made substantial contributions to the conception and design of the study, acquisition of data, analysis and interpretation of the data, and drafting of the manuscript. Ahmad R Soboh and Dima Sadi have made substantial contributions to the conception and design of the study, the acquisition of data, the analysis and interpretation of the data, and the drafting of the manuscript. Deyab R Khloof and Ammar A Aghbar have made substantial contributions to the conception and design of the study, the acquisition of data, the analysis and interpretation of the data, and the drafting of the manuscript. All authors have given final approval of the version to be published.

\section{ACKNOWLedgments}

The authors would like to thank all participants without whom there would be no study.

\section{References}

1. Rabinowitz YS. Keratoconus. Surv Ophthalmol 1998;42(4):297-319. DOI: 10.1016/S0039-6257(97)00119-7.

2. Romero-Jimenez M, Santodomingo-Rubido J, Wolffsohn JS. Keratoconus: a review. Cont Lens Anterior Eye 2010;33(4):157-166. DOI: 10.1016/j.clae.2010.04.006.

3. Wollensak G, Spoerl E, Seiler T. Riboflavin/ultraviolet-a-induced collagen crosslinking for the treatment of keratoconus. Am J Ophthalmol 2003;135(5):620-627. DOI: 10.1016/S0002-9394(02) 02220-1.

4. Kymionis GD, Grentzelos MA, Kankariya VP, et al. Long-term results of combined transepithelial phototherapeutic keratectomy and corneal collagen crosslinking for keratoconus: Cretan protocol. J Cataract Refract Surg 2014;40(9):1439-1445. DOI: 10.1016/j.jcrs.2014.01.040.

5. Kanellopoulos AJ, Binder PS. Collagen cross-linking (CCL) with sequential topography-guided PRK: a temporizing alternative for keratoconus to penetrating keratoplasty. Cornea 2007;26(7):891-895. DOI: 10.1097/ICO.0b013e318074e424.

6. Kymionis GD, Grentzelos MA, Portaliou DM, et al. Corneal collagen cross-linking $(C X L)$ combined with refractive procedures for the treatment of corneal ectatic disorders: CXL plus. J Refract Surg 2014;30(8):566-576. DOI: 10.3928/1081597X-20140711-10.

7. Kymionis GD, Portaliou DM, Kounis GA, et al. Simultaneous topography-guided photorefractive keratectomy followed by corneal collagen cross-linking for keratoconus. Am J Ophthalmol 2011;152(5):748-755. DOI: 10.1016/j.ajo.2011.04.033.

8. Stojanovic A, Zhang J, Chen X, et al. Topography-guided transepithelial surface ablation followed by corneal collagen crosslinking performed in a single combined procedure for the treatment of keratoconus and pellucid marginal degeneration. J Refract Surg 2010;26(2):145-152. DOI: 10.3928/1081597X-20100121-10.

9. Tuwairqi WS, Sinjab MM. Safety and efficacy of simultaneous corneal collagen cross-linking with topography-guided PRK in managing low-grade keratoconus: 1-year follow-up. J Refract Surg 2012;28(5):341-345. DOI: 10.3928/1081597X-20120316-01.

10. Lin DT, Holland S, Tan JC, et al. Clinical results of topography-based customized ablations in highly aberrated eyes and keratoconus/ ectasia with cross-linking. J Refract Surg 2012;28(11 Suppl):S841-S848. DOI: 10.3928/1081597X-20121005-06.

11. Alessio G, L'Abbate M, Sborgia C, et al. Photorefractive keratectomy followed by cross-linking versus cross-linking alone for management 
of progressive keratoconus: two-year follow-up. Am J Ophthalmol 2013;155(1):54-65. DOI: 10.1016/j.ajo.2012.07.004.

12. Greenstein SA, Shah VP, Fry KL, et al. Corneal thickness changes after corneal collagen crosslinking for keratoconus and corneal ectasia: one-year results. J Cataract Refract Surg 2011;37(4):691-700. DOI: 10.1016/j.jcrs.2010.10.052.

13. Mazzotta C, Balestrazzi A, Baiocchi S, et al. Stromal haze after combined riboflavin-UVA corneal collagen cross-linking in keratoconus: in vivo confocal microscopic evaluation. Clin Exp Ophthalmol 2007;35(6):580-582. DOI: 10.1111/j.1442-9071.2007. 01536.x.

14. Mencucci R, Paladini I, Virgili G, et al. Corneal thickness measurements using time-domain anterior segment OCT, ultrasound, and Scheimpflug tomographer pachymetry before and after corneal cross-linking for keratoconus. J Refract Surg 2012;28(8):562-566. DOI: 10.3928/1081597X-20120703-02. 